\title{
Assessment of Human Hemodynamics under Hyper- and Microgravity: Results of two Aachen University Parabolic Flight Experiments
}

\author{
N. Blanik, M. Hülsbusch, M. Herzog, C. R. Blazek
}

Astronauts complain about fluid shifts from their lower extremities to their head caused by weightlessness during their flight into space. For a study of this phenomenon, RWTH Aachen University and Charité University Berlin participated in a joint project on two parabolic flight campaigns of the German Aerospace Centre (DLR) in September 2005 and June 2006. During these campaigns, the characteristics of the rapid fluid shifts during hyper-and micro gravity were measured by a combination of PPG and PPGI optoelectronic sensor concepts.

Keywords: rapid fluid shifts, photoplethysmography, photoplethysmography imaging, parabolic flights, microgravity.

\section{Introduction}

During manned flights into space fluid shifts from the lower extremities to the heads of the astronauts have been detected. This phenomenon is termed "puffy face syndrome" or "bird legs", because of the optical impression of the subject (bloated face and thin legs). These effects are caused by the unfamiliar influence of microgravity on the human body. The fluid shifts into the head result in motion sickness, concentration problems, vision degradation and other potentially dangerous symptoms during space flights. Under normal conditions (on earth), gravitation arranges a fluid balance in the human body. Thereby most of the blood volume is allocated to the veins of the lower extremities. Figure 1 shows the distribution of the blood volume inside the human body while standing on earth. Flying into space and escaping from the gravitational force disturbs this balance. Especially interesting in this kind of fluid shifts are the dynamic characteristics of the blood flow immediately after the moment when the gravity changes. So far these phenomena are only known qualitatively.

To research these dynamic behaviors, the subjects were exposed to a rapid change of gravity (between zero and

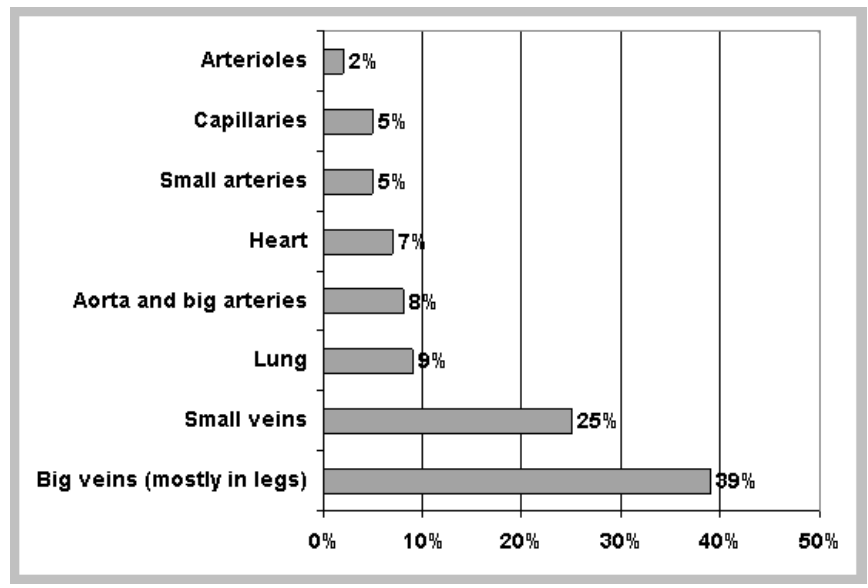

Fig. 1: Distribution of blood volume for the different parts of the vascular system
1.8 times the normal gravitational force on earth, i.e. $0 \mathrm{~g}$ and $1.8 \mathrm{~g}$, respectively) on parabolic flights. During this time, sensors measured the relative change of the blood volume in the upper skin layers of the legs and forehead.

\section{Parabolic flight}

Studies on gravitational effects requiring lower than normal gravitational force on earth are impossible under usual laboratory conditions. There are only a few ways to eliminate the disturbing influence of gravity on earth: free fall towers, ballistic rockets, parabolic flights or just leaving the earth. Parabolic flights can be taken on board the Airbus A300 ZERO-G owned by the European Space Agency (ESA). Each year the Germany Aerospace Centre (DLR) organizes parabolic flight campaigns especially for research projects.

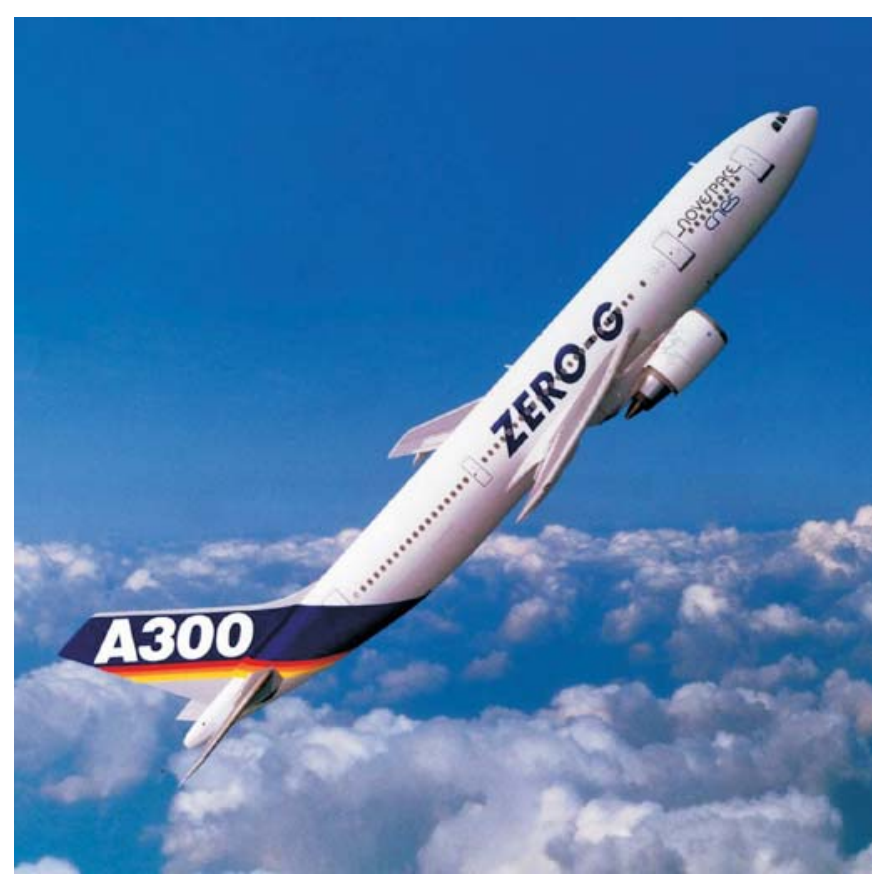

Fig. 2: Airbus A300 Zero-G directly before injecting the $0 \mathrm{~g}$-phase 


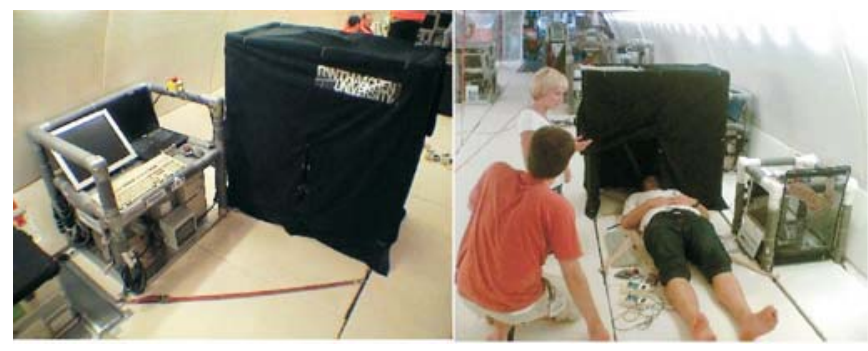

Fig. 3: Experimental configuration on the $8^{\text {th }}$ DLR parabolic flight campaign

In September 2005 and June 2006, our joint research program had the opportunity to fly our experiments on the $7^{\text {th }}$ and $8^{\text {th }}$ DLR parabolic flight campaigns in Bordeaux and Cologne, respectively. For this purpose the specially prepared Airbus A300 is equipped with up to twelve separate experiments conducted by various participants. The campaign comprises 4 to 5 flight days. During one flight the aeroplane accomplishes 31 parabola maneuvers. These flight maneuvers (Fig. 4) follow a special trajectory which results in micro-

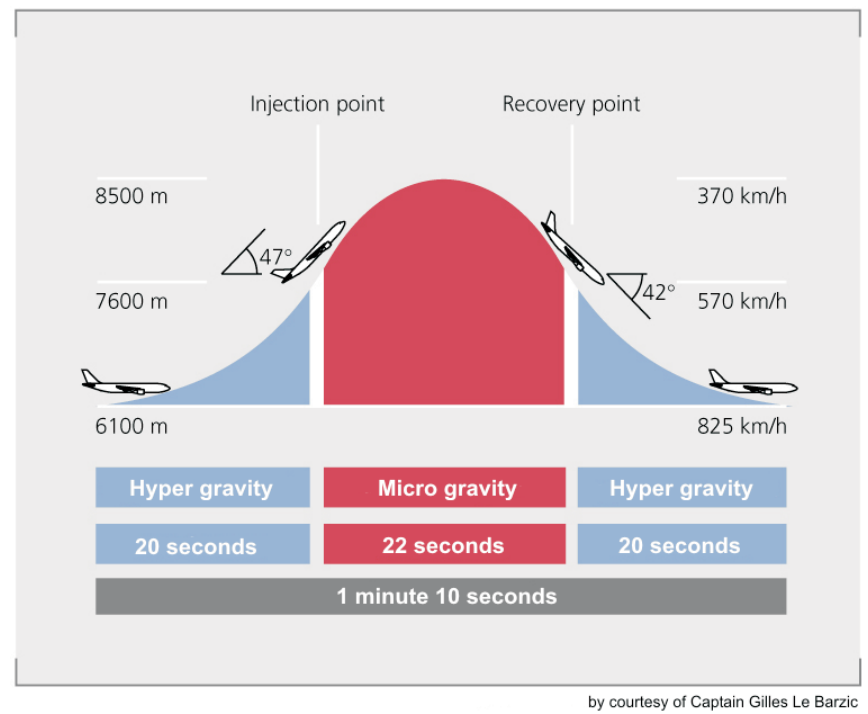

Fig. 4: Flight phases of parabolic flight

gravity up to 22 seconds (Fig. 5) inside the aircraft. One maneuver can be separated into 3 phases. In the first one, the plane is pulled up from the horizontal to a climbing flight up to an angle of $47^{\circ}$. During this time (about 20 seconds) the occupants of the plane are exposed to $1.8 \mathrm{~g}$. In the next phase the state of microgravity is achieved. Therefore the plane is steered in a ballistic trajectory, a parabola. Inside the plane a reduced gravity of about $0.02 \mathrm{~g}$ can be achieved. This lasts

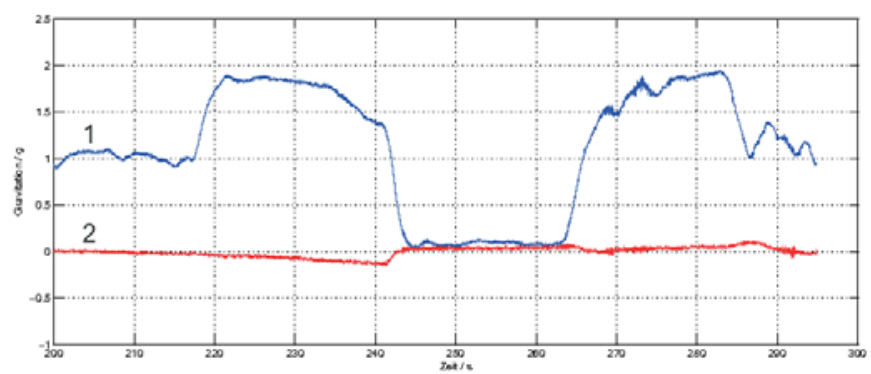

Fig. 5: Typical development of g-forces during a parabola maneuver (1: g-forces vertically, 2: g-forces in flight direction) another 20 seconds. After that in the third phase the plane is pulled out to its normal flight level. In doing this $1.8 \mathrm{~g}$ is achieved for another 20 seconds. After a break of a few minutes the maneuver is repeated.

\section{Optoelectronic sensors}

Optoelectronic sensor concepts provide a good opportunity to assess the blood volume in the skin. They are basically non-invasive and thus allow measurements also under space conditions and provide an unproblematic way to obtain data from the subjects or even to exchange subjects, during flight in the time between two parabolas. A greater number of subjects can therefore be measured.

\subsection{Photoplethysmography sensor}

The first sensor type used here is the photopletysmography (PPG) sensor. This sensor is stuck to the surface of the skin. It contains a monochromatic light source and a photo detector. It uses the fact that blood absorb light at a higher rate than the surrounding tissue. The backscattered or reflected light intensity in well perfused tissues is lower than in tissues with lower perfusion. The scattered light is received by the detector, and conclusions are drawn from the perfusion of the skin in the area below the sensor. The achievable penetration depth lies between one and three millimeters, depending on the wavelength of the light source that is used.

\subsection{Photoplethysmography imaging}

Photopletysmography imaging (PPGI) is an advancement of classical PPG. It uses the same underlying circumstances. In contrast to the PPG-sensor, an external light source and a high sensitive camera are used. Like the PPG-sensor, this camera is also able to detect in its view small changes of light intensity caused by the shifting of blood in the skin. Using a camera as detector, the analysis of the blood volume shift is not limited to a single spot but measures a larger part of the skin surface. An additional benefit is the contactless measurement principle.

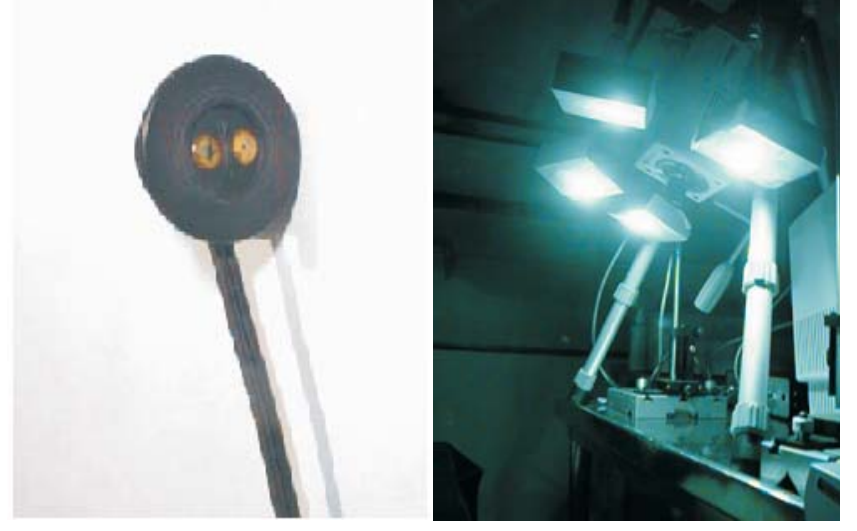

Fig. 6: PPG sensor (left) and PPGI camera with surrounding light sources (right)

\section{Results}

After the flights, the data from the different subjects were analysed and it was first ascertained that fluid shifts occurred to all subjects. This proves that even the short time span of 
about 20 seconds of a single parabola suffices for clear detection. Furthermore, a clear shift from the lower body parts to the head could be verified, as had been predicted.
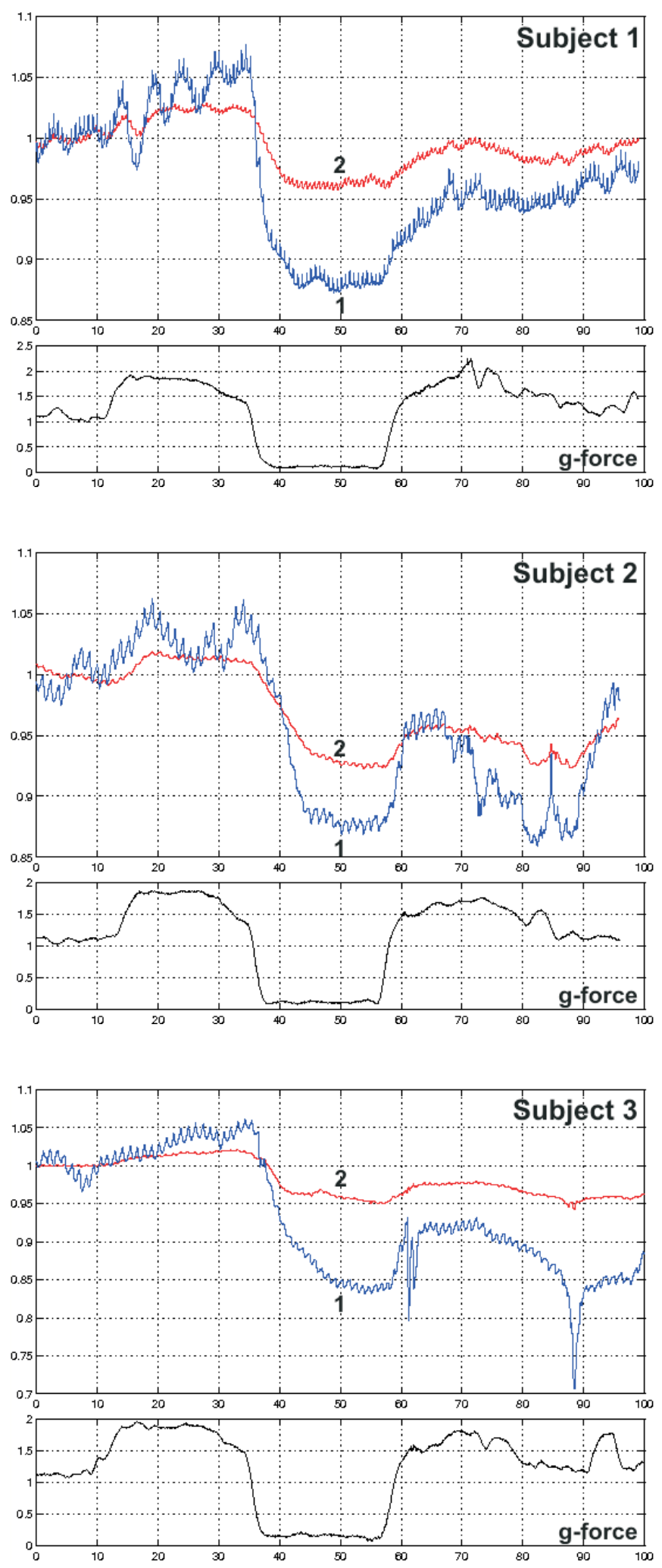

Fig. 7: Different kinetics of blood volume shifts of three subjects (measuring points on the forehead using a green (1) and an infrared light source (2), normalized at start of measurement)
Fig. 7 shows three typical PPG measurements. They contain the characteristics of one subject each. These recordings were taken with sensors working with green and infrared light sources. They are placed on the subjects' foreheads. The detected light intensity is displayed in curve 1 (green light) and curve 2 (infrared light). The lower black curve shows the development of the $\mathrm{g}$-forces during the parabola. The small and fast oscillations seen in the curves are caused by the subjects' heartbeats (clearly visible in the second blue curve) and breathing. However, the main focus of this research program was on the characteristics of the low-frequency components.

It is clear to see that immediately after the injection of zero gravity the detected backscattered light intensity declines rapidly. This corresponds to an increasing volume of blood in the skin under the sensor.

A comparison of the data for the different subjects shows that all of them had a very quick reaction to the altered gravitational force. The time span between the change of gravity and the minimum in the detected light intensity (or the maximum if measured at the legs) was very short (only a few seconds). However, this also differs among the subjects. These individual characteristics are detectable with all types of sensors used here. A statistical analysis of the data is shown in Fig. 8. It shows the relative shift of the light intensity detected by the sensor during the $0 \mathrm{~g}$-phase in contrast to the start of the parabola maneuver. The differences in the range of different light colors are caused by the individual penetration depth of the light wave length that is used.

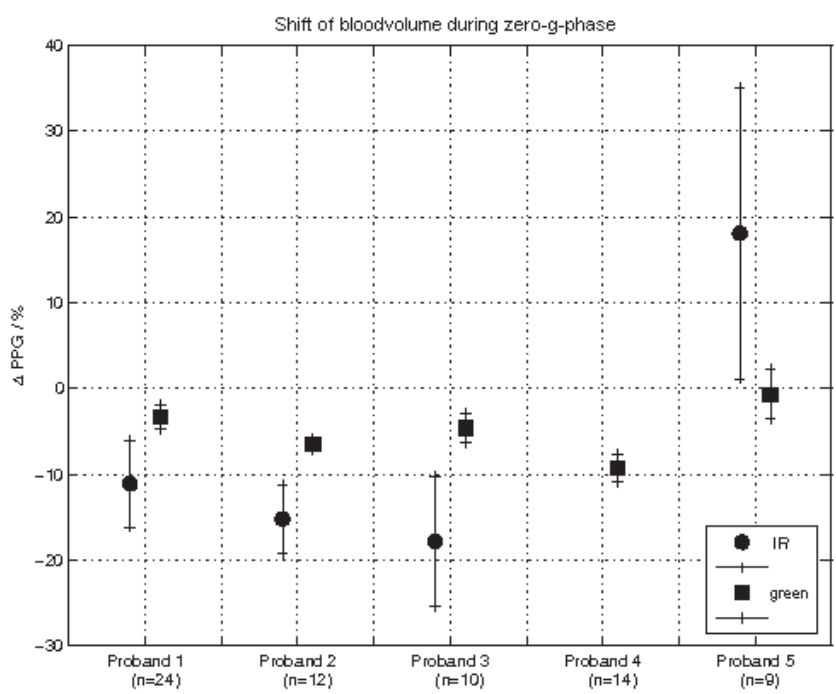

Fig. 8: Statistical analysis of skin blood volume changes measured with PPG sensors on subjects' foreheads

An additional observation of the time that the blood takes to flow out of the vessels in the leg (during a change of gravity from $1.8 \mathrm{~g}$ to $0 \mathrm{~g}$ ) with the time it takes to flow back (during change from $0 \mathrm{~g}$ to $1.8 \mathrm{~g}$ ) shows that the former time span is noticeably shorter than the latter one. An explanation for this is that under microgravity conditions the resistance for the blood flow out of the leg vessels towards the heart (and head) is very little. In addition this flow is assisted by the venous valves. Healthy venous valves prevent a direct flow backwards through the veins. The flow towards the legs is thus limited to the heart pumping volume and the arterial blood flow. 


\section{Acknowledgments}

The experiment was conducted in cooperation with the Centre for Space Medicine, Department of Physiology, Faculty of Medicine of the Charité University, Berlin (Prof. Gunga, principal investigator) and the Institute of High Frequency Technology, RWTH Aachen (Prof. Blazek).

\section{References}

[1] Blazek, V.: Optoelektronische Systemkonzepte für nichtinvasive Kreislaufdiagnostik,, Optoelek Magazin, 1991, p. 212-219.

[2] Space Travel and the Effects of Weightlessness on the Human Body, Canadian Space Agency. http://www.space.gc.ca/ asc/pdf/educator-microgravity_science_stu.pdf

[3] Golenhofen, K.: Physiologie. Urban und Schwarzenberg Verlag, München 1997.

[4] Blazek, V., Schultz-Ehrenburg, U. (Eds.): Quantitative Photoplethysmography - Basic Facts and Examination Tests for Evaluating Peripheral Vascular Functions, VDI Verlag, Düsseldorf 1996.
[5] Blazek, V.: Funktionelle Beinvenendiagnostik mit der quantitativen, digitalen Photoplethysmographie, Viavital Verlag, Essen 2005, p. 49-58.

Nikolai Blanik

e-mail: blanik@ihf.rwth-aachen.de

Markus Hülsbusch

e-mail: huelsbusch@ihf.rwth-aachen.de

Markus Herzog

e-mail: herzog@ihf.rwth-aachen.de

Institute of High Frequency Technology

RWTH Aachen University

Germany

Claudia R. Blazek

e-mail: claudia.blazek@gmx.de

Department of Dermatology

Medical Faculty

RWTH Aachen University, Germany 